\title{
Simulação da distribuição de água em diferentes condições de vento e espaçamentos entre aspersores
}

\author{
Lessandro C. Faria ${ }^{1}$, Giuliani do Prado ${ }^{2}$, Alberto Colombo ${ }^{3}$, \\ Henrique F. E. de Oliveira ${ }^{4}$ \& Samuel Beskow ${ }^{1}$
}

\begin{abstract}
RESUMO
Existem inúmeros fatores que afetam a uniformidade de distribuição de água pelos aspersores como, por exemplo, o tipo de aspersor e suas condições operacionais, o espaçamento e sua disposição no campo e a velocidade e direção do vento. Se os aspersores forem selecionados e utilizados adequadamente considerando-se os fatores supracitados, eles podem proporcionar maiores uniformidades de distribuição de água e menores custos do sistema de irrigação. Como a avaliação de todos esses fatores é uma tarefa complexa e morosa, a simulação computacional tem sido uma ferramenta útil no dimensionamento de sistemas de irrigação. Neste estudo objetivou-se determinar, com auxílio de simulação computacional, a uniformidade de distribuição de água do canhão hidráulico Plona-RL250, operando em diferentes condições de vento e espaçamentos e disposições de aspersores. Observou-se que a direção do vento exerceu pouca influência sobre os valores de CUC. Nenhuma vantagem foi encontrada para disposição triangular em comparação com a disposição retangular de aspersores, exceto quando foi usado um espaçamento menor que $40 \%$ do diâmetro molhado pelo canhão hidráulico, ao longo da linha principal.
\end{abstract}

Palavras-chave: irrigação, canhão hidráulico, modelo semiempírico

\section{Simulation of water distribution under different conditions of wind and spacing between sprinklers}

\begin{abstract}
There are various factors that affect water distribution uniformity from sprinklers, such as sprinkler type and its operational conditions, spacing and sprinkler setup in the field and wind speed and direction. If sprinklers are selected and used appropriately considering the aforementioned factors, they can result in higher water distribution uniformities and lower costs. Since the evaluation of all the above-mentioned factors is a complex and time-consuming task, computational simulation is a useful tool for the designing of irrigation systems. This study had as objective to determine, with the aid of computational simulation, the water distribution uniformity of the Plona-RL250 gun sprinkler working under different wind conditions as well as spacing and layouts of gun sprinklers. It was observed that wind direction exerted little influence on the water distribution uniformity values. No advantage was found for the triangular spacing in comparison with the rectangular spacing, except when a spacing less than $40 \%$ of the wetted diameter by the sprinkler was used along the main pipeline.
\end{abstract}

Key words: irrigation, gun sprinkler, semi-empirical model 


\section{INTRODUÇÃO}

A busca por novas estratégias para otimização do uso de recursos hídricos na agricultura tem sido ferramenta de diversos estudos. O Brasil apresenta regiões com déficit hídrico e distribuições irregulares de chuva durante todo o ano; desta forma, um planejamento da irrigação com seleção adequada de aspersores e suas condições operacionais, tal como espaçamentos e disposições no campo que proporcionem maiores uniformidades de distribuição de água e menores custos de investimento do sistema, são de grande importância para a sustentabilidade da agricultura irrigada no país.

Diversos fatores afetam a uniformidade de distribuição de água de aspersores dentre eles a forma geométrica do perfil radial de distribuição de água (dada pela combinação de bocais, pressão de serviço e ângulo de saída do jato d'água) o espaçamento entre aspersores, a disposição dos aspersores no campo (Keller \& Bliesner, 1990) e a velocidade e direção do vento em relação à linha lateral de aspersores (Tarjuelo et al., 1999). Conforme Seginer et al. (1991) na irrigação por aspersão o padrão de distribuição de água é bastante afetado pelo vento tendo, como consequência, um recebimento insuficiente de água em determinados pontos da área irrigada.

Avaliando alguns desses fatores para sistemas convencionais de irrigação equipados com canhão hidráulico, Azevedo et al. (2000) constataram que a velocidade do vento foi o fator que mais prejudicou a uniformidade de distribuição de água do aspersor; resultados similares foram observados por Vories \& Bernuth (1986) e Tarjuelo et al. (1999).

A melhor disposição (quadrada, retangular e triangular) entre aspersores é bastante discutida na literatura. Alguns estudos recomendam disposição triangular entre aspersores enquanto outros não demonstram vantagens sobre a disposição retangular (Tarjuelo et al., 1999); entretanto, Keller \& Bliesner (1990) afirmam que a melhor disposição dependerá do perfil de distribuição de água do aspersor. De acordo com Bernardo et al. (2006) as disposições retangulares e quadradas são mais usuais sendo que os espaçamentos, a disposição e a pressão de serviço dos aspersores devem ser constantes para manter a uniformidade sobre toda a área irrigada.

Na seleção de espaçamento e disposição de aspersores devese considerar a melhor relação entre os custos do sistema e os benefícios associados à uniformidade de distribuição de água obtendo-se o maior retorno econômico da irrigação; assim, torna-se imprescindível prever, de forma rápida e precisa a uniformidade de sistemas convencionais antes mesmo de sua implantação no campo.

De acordo com Chen \& Wallender (1984) sistemas com espaçamentos reduzidos entre aspersores geralmente possuem maiores uniformidades de distribuição de água porém seus custos e as taxas de aplicação de água são maiores. Os autores apresentam superfícies de resposta com valores de uniformidade de distribuição de água para os perfis clássicos de Christiansen (1942) em função do espaçamento entre aspersores, expressos em função do diâmetro molhado dos mesmos para as disposições retangulares e triangulares. Tais superfícies de respostas auxiliam na determinação de melhores disposições e espaçamentos entre aspersores na linha lateral e entre elas.
Uma maneira de determinar a uniformidade de distribuição de água de aspersores operando em diferentes espaçamentos e disposições (quadrada, retangular e triangular) é por meio de simulações computacionais visto que, em condições de campo, avaliar as inúmeras possibilidades de combinações entre os fatores que as afetam seria tarefa complexa e morosa.

Diferentes modelos matemáticos têm sido utilizados para simular a distribuição de água de aspersores operando em condições de vento. Apesar da ampla utilização de modelos físicos baseados na teoria balística para simular a uniformidade de distribuição de água de aspersores, atualmente diversos autores (Smith et al., 2008; Faria et al., 2009; Prado \& Colombo, 2010a; 2010b; Vellame et al., 2011) têm utilizado modelos empíricos de simulação devido principalmente ao reduzido número de parâmetros de ajuste destacando-se, entre eles, o modelo semiempírico de Richards \& Weatherhead (1993).

Para uma análise mais ampla dos efeitos do vento sobre o padrão de distribuição de água e as inúmeras condições operacionais de aspersores, a modelagem matemática se faz necessária. Desta maneira, este estudo teve como objetivo determinar, com auxílio de simulações computacionais, a uniformidade de distribuição de água do canhão hidráulico Plona-RL250, operando em diferentes condições de velocidade e direção de vento e diferentes espaçamentos e disposições entre aspersores.

\section{Material e Métodos}

Na condução do trabalho foram utilizados os dados técnicos (vazão, raio de alcance e perfil radial de distribuição de água) de um aspersor tipo canhão hidráulico de reversão lenta PlonaRL250, que apresenta ângulo de lançamento do jato de água de $24^{\circ}$, flange de acoplamento de $2 \frac{1}{2}$ " e giro proporcionado pelo impacto de um de seus dois braços oscilantes. Este aspersor opera com dois bocais cônicos convergentes, um principal, disponível em oito diâmetros $(14,16,18,20,22,24,26,28$ $\mathrm{mm})$ e um auxiliar, disponível em três diâmetros $(5,6$ e $7 \mathrm{~mm})$ além de cinco valores de pressão de serviço $(294,392,490$, 580 e $686 \mathrm{kPa}$ ).

Todas as prováveis combinações entre 8 bocais principais, 5 pressões de serviço, 3 bocais auxiliares e ainda a possibilidade de operar sem bocal auxiliar, possibilitam a formação de 160 perfis radiais de distribuição de água do canhão hidráulico Plona-RL250. Sendo assim, com o auxílio do aplicativo SimulaSoft (Prado \& Colombo, 2010a) obtiveram-se os 160 perfis radiais de distribuição de água canhão Plona-RL250 utilizados neste estudo.

Para cada condição operacional do canhão Plona-RL250 foram obtidos, com o SimulaSoft, os dados de vazão, raio de alcance e o perfil radial de distribuição de água do aspersor em condições de ausência de vento. Cada perfil radial de distribuição de água é composto de um conjunto contendo 20 valores de intensidade de aplicação de água correspondentes às mesmas 20 posições em relação ao aspersor (que variam desde $2,5 \%$ até $97,5 \%$ do raio de alcance do canhão hidráulico, em intervalos regulares de 5\%).

Conforme Solomon \& Bezdek (1980) para cada conjunto de perfil radial de distribuição de água as distâncias ao aspersor 
foram expressas em termos da fração do raio de alcance e os valores de intensidade de aplicação de água foram expressos em termos da fração da intensidade média de aplicação de água do aspersor.

Os 160 conjuntos de perfis radiais adimensionais foram submetidos à análise de agrupamento visando identificar grupos de condições operacionais (pressão vs. bocais) que resultem em perfis adimensionais com valores muito semelhantes e possam ser representados por um mesmo perfil típico. Seguindo recomendações de Solomon \& Bezdek (1980) realizou-se a análise de agrupamento dos perfis adimensionais com o auxílio do algoritmo "K-Means" (Tou \& Gonzales, 1974) de acordo com o descrito por Faria (2008) e Colombo et al. (2009).

Para simular a distribuição espacial de água de um aspersor operando isoladamente em diferentes condições de vento, uma rotina computacional foi desenvolvida em Visual Basic para Aplicativos (VBA) da planilha eletrônica Excel baseada no modelo semiempírico de Richards \& Weatherhead (1993). Este modelo foi ajustado para o aspersor Plona-RL250 conforme descrito por Colombo et al. (2009) e validado para sistemas convencionais de irrigação, por Faria et al. (2009).

De acordo com Faria et al. (2009) a rotina desenvolvida gera os valores de precipitação $\left(\mathrm{mm} \mathrm{h}^{-1}\right)$ ao longo de uma malha de pontos (coletores fictícios) uniformemente distribuídos ao redor do canhão hidráulico Plona-RL250 observando-se o mesmo arranjo dos coletores utilizados nos ensaios de campo (Figura 1).

Para efeito das simulações consideraram-se os seguintes valores de entrada:

- Perfis radiais de distribuição de água do canhão PlonaRL250 operando na ausência de vento, nas 160 diferentes condições operacionais do aspersor.

- Velocidade média do vento (V): $0 ; 1,4 ; 2,8$ e $4,2 \mathrm{~m} \mathrm{~s}^{-1}$.

- Direção média do vento $(\Phi): 0^{\circ}$ (paralelo à linha lateral), $45^{\circ}$ e $90^{\circ}$ com relação à linha lateral (perpendicular), Figura 1.

- Coordenadas (x,y) dos coletores tomadas no sistema de eixos orientados dos ensaios de campo.

- Ângulo de saída do jato de água do canhão Plona-RL250 em relação ao plano horizontal $\left(\mathrm{e}=24^{\circ}\right)$.

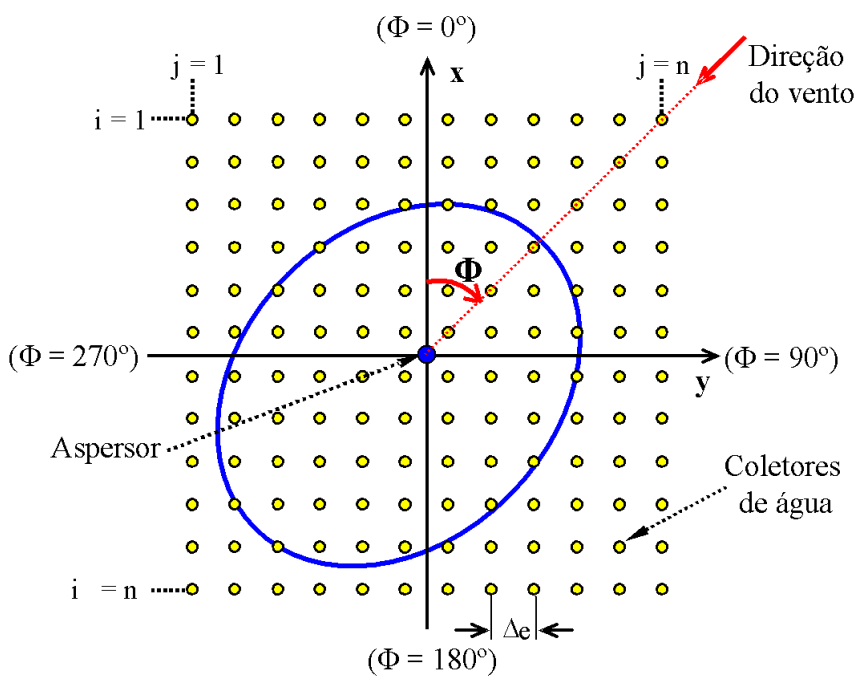

Figura 1. Esquema do sistema de eixos $(x, y)$ utilizado nos ensaios de campo e ângulo $(\Phi)$ formado entre $o$ eixo $\mathrm{x}$ e o vetor vento
- Valores dimensionalizados ajustados por Faria (2008) para o aspersor Plona-RL250, das constantes empíricas do modelo de Richards \& Weatherhead (1993): $A=1,7 ; B=0,6 ; C=-0,5$; $\mathrm{D}=0,1 ; \mathrm{E}=19,1$ e $\mathrm{F}=-16,0$.

$\mathrm{Na}$ determinação da uniformidade de distribuição de água simulada em diferentes situações em que se considerou espaçamento regular entre coletores $(\Delta \mathrm{e})$ de $3 \mathrm{~m}$ e espaçamento entre aspersores múltiplos de $6 \mathrm{~m}$ variando de 10 até $100 \%$ do diâmetro molhado do aspersor, as distribuições espaciais simuladas para cada condição de vento foram sobrepostas. No processo de sobreposição foram considerados os aspersores dispostos em arranjos retangulares e triangulares.

A uniformidade de distribuição de água dos valores sobrepostos foi quantificada por meio do coeficiente de uniformidade de Christiansen - CUC (Christiansen, 1942) e os valores de CUC obtidos foram agrupados em diferentes superfícies de respostas que representavam os perfis radiais típicos do canhão hidráulico Plona-RL250 que, por sua vez, foram indicados na análise de agrupamento realizada. Nessas superfícies os valores de CUC, para as disposições retangulares e triangulares, foram apresentados em função dos espaçamentos entre aspersores, na linha lateral ( $\mathrm{Sl})$ e na linha principal (Sp), expressos como fração do diâmetro molhado do aspersor (\% DM).

Em cada superfície de resposta foi traçada a curva que delimita as combinações de espaçamentos a partir das quais a sobreposição das áreas molhadas pelos aspersores é incompleta, ou seja, ocorre o aparecimento de áreas secas na ausência de vento. As combinações de espaçamentos entre aspersores que delimitam a região a partir da qual ocorre o comprometimento da cobertura completa da área irrigada, foram determinadas para os arranjos retangulares e triangulares por meio das Eqs. 1 e 2, respectivamente (Prado et al., 2008).

$$
\begin{gathered}
\frac{\mathrm{S} l}{\mathrm{DM}}=\sqrt{1-\left(\frac{\mathrm{Sp}}{\mathrm{DM}}\right)^{2}} \\
\frac{\mathrm{Sl}}{\mathrm{DM}}=\sqrt{1-4 \cdot\left(\frac{\mathrm{Sp}}{\mathrm{DM}}-0,5\right)^{2}}
\end{gathered}
$$

\section{Resultados E Discussão}

A análise de agrupamento dos 160 perfis radiais adimensionais de distribuição de água pelo algoritmo de agrupamento "K-Means" (Tou \& Gonzales, 1974) indicou que quatro perfis radiais típicos (I, II, III e IV) mostrados na Figura 2, são suficientes para representar todas as prováveis combinações operacionais de bocais e pressões de serviço do canhão Plona-RL250. Observa-se, na Figura 2, a ocorrência do acúmulo de aplicação de água na extremidade final do perfil adimensional correspondente ao grupo I, que se dissipa à medida em que se passa para os grupos II, III e IV; referido comportamento do perfil radial de distribuição de água, em função do aumento da pressão, está de acordo com o previsto por Christiansen (1942), Keller \& Bliesner (1990) e Bernardo et al. (2006). 

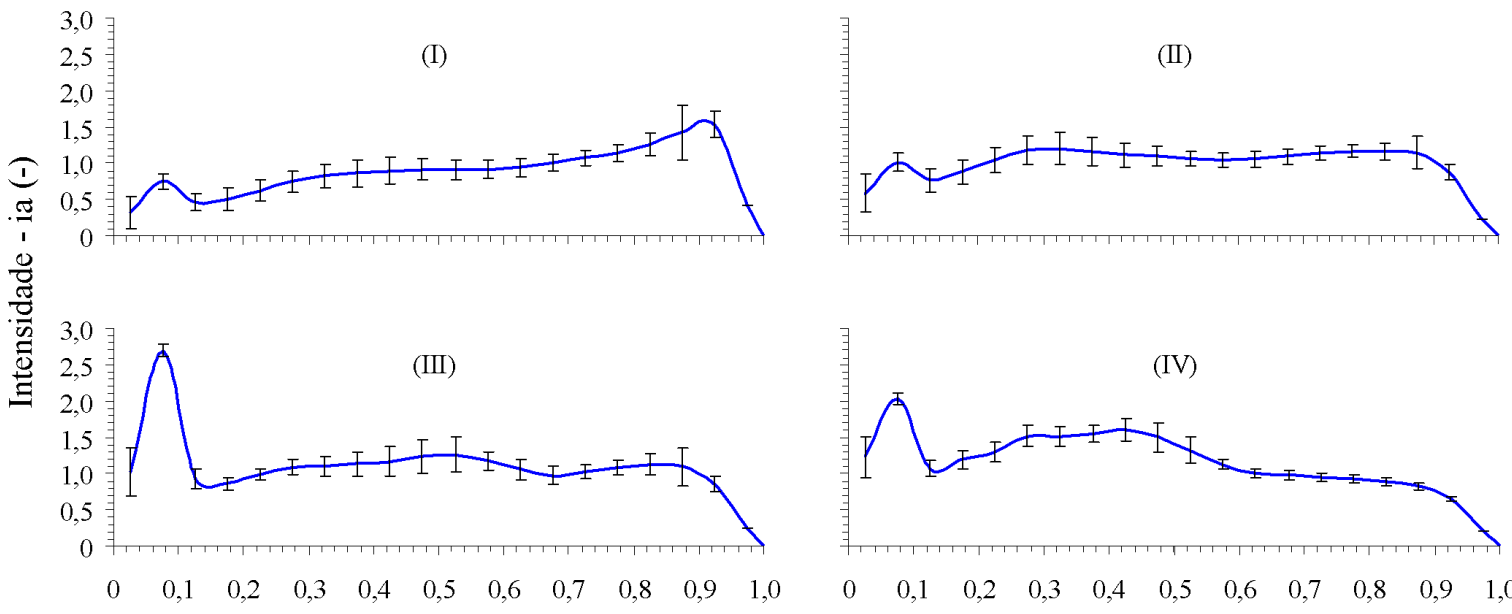

Distância ao aspersor - ra (-)

Figura 2. Perfis adimensionais I, II, III e IV do canhão Plona-RL250; as barras verticais representam a variação de comprimento equivalente $\mathrm{a} \pm 1$ desvio padrão

Na Figura 3 se apresenta o diâmetro molhado pelo aspersor para as condições operacionais (bocais $v s$. pressão de serviço) que determinam diferentes níveis de pulverização do jato de água do canhão Plona-RL250 para os quatro perfis típicos considerados (Figura 2).

De acordo com a Figura 3, a região de ocorrência de cada um dos quatro grupos obtidos na análise de agrupamento pode ser associada à ocorrência de condições operacionais que determinam diferentes níveis de pulverização do jato de água do aspersor. A parte inferior da direita dessas superfícies, onde predomina o perfil I, corresponde à combinação dos maiores bocais com as menores pressões de serviço que, de acordo com Keller \& Bliesner (1990) resultam em gotas maiores. O perfil típico IV corresponde a condições inversas ao perfil I, ou seja,

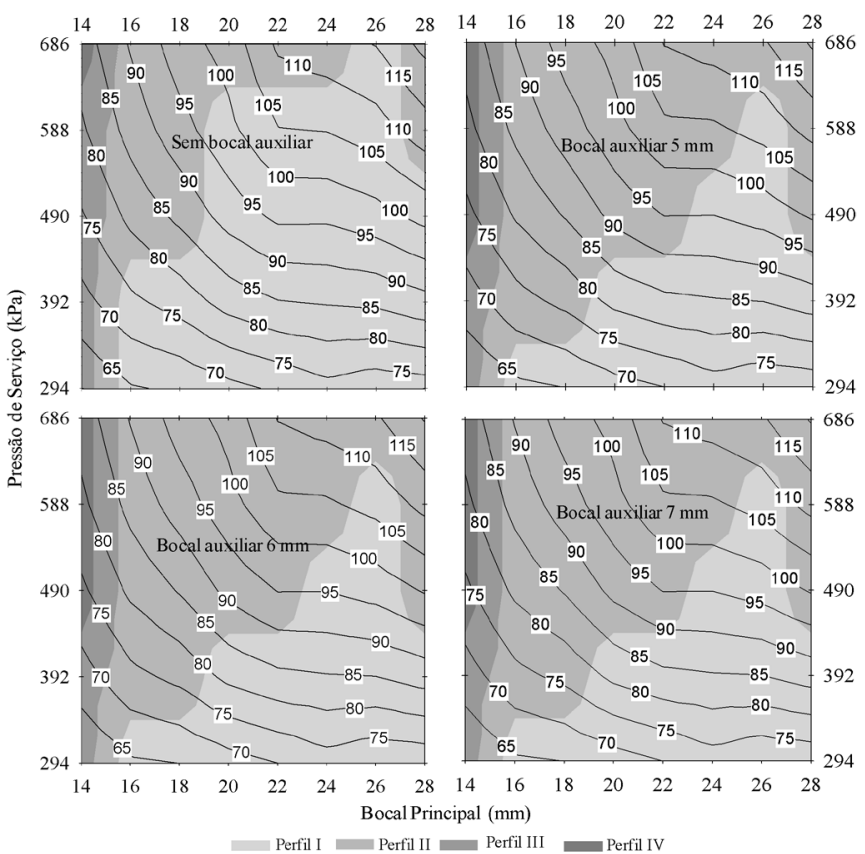

Figura 3. Diâmetro molhado (m) e grupo característico (I, II, III e IV) correspondente às diferentes combinações entre pressão e diâmetro dos bocais do canhão PlonaRL250 maiores pressões e menores bocais, que resultam em gotas de menor diâmetro; os perfis típicos II e III correspondem a condições intermediárias entre os perfis I e IV.

Conforme Prado et al. (2008) as condições operacionais que geram um pico de aplicação de água na extremidade final do perfil radial de distribuição de água (perfil típico I) decorrentes de combinações de baixas pressões de serviço e bocais maiores, devem ser evitadas. De acordo com os autores, na utilização das condições operacionais que resultam no perfil I (Figura 2) índices adequados de uniformidade de distribuição de água são alcançados com combinações de espaçamentos entre aspersores (na linha lateral e entre linhas laterais) inferiores a $40 \%$ do diâmetro molhado pelo aspersor; assim, não devem ser utilizados em condições práticas.

Para os demais perfis adimensionais de distribuição de água obtidos na análise de agrupamento (perfis II, III e IV) foram geradas superfícies de respostas contendo valores simulados de coeficientes de uniformidade de Christiansen (CUC) para sistemas convencionais de irrigação com aspersores dispostos em arranjos retangulares e triangulares operando em diferentes condições de vento.

Na Figura 4 são mostrados os valores de CUC simulados na ausência de vento para sistemas convencionais de irrigação em diferentes espaçamentos entre canhões Plona-RL250, em função da porcentagem do diâmetro molhado (\% DM) e condições operacionais referentes aos perfis típicos II, III e IV para disposição retangular e triangular entre aspersores. Nesta figura a linha pontilhada delimita as combinações de espaçamentos a partir das quais a sobreposição das áreas molhadas pelos aspersores adjacentes é incompleta, ou seja, as combinações de espaçamentos abaixo da linha pontilhada garantem um mínimo de sobreposição.

As superfícies de resposta da Figura 4 são semelhantes às apresentadas por Chen \& Wallender (1984) para os perfis D e E descritos por Christiansen (1942). Nas superfícies de resposta (Figura 4) que indicam espaçamento retangular entre aspersores observa-se uma simetria obtida ao longo de uma linha imaginária de $45^{\circ}(\mathrm{Sl}=\mathrm{Sp})$ que serve para ilustrar a exatidão da rotina computacional utilizada para sobreposição dos padrões 


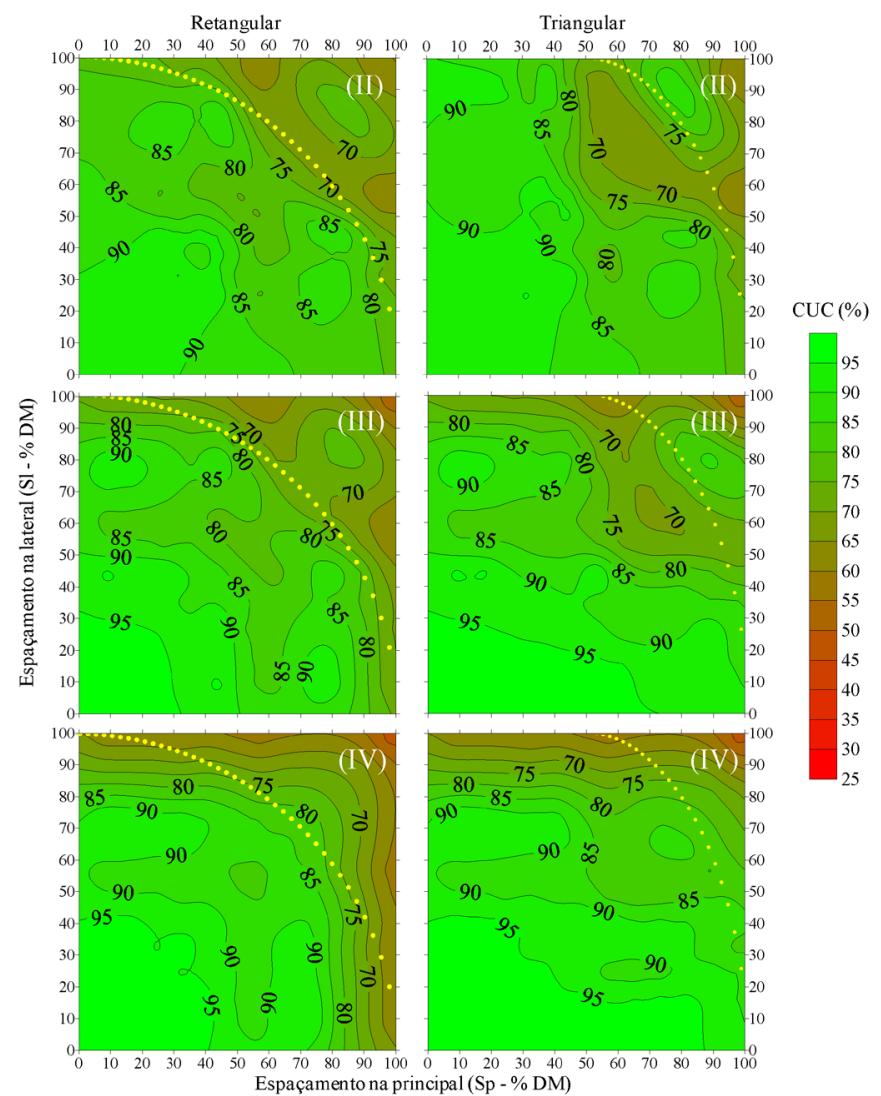

Figura 4. Valores de coeficiente de Christiansen CUC (\%) em função do espaçamento entre aspersores (\% DM) e condições operacionais referentes aos perfis típicos II, III e IV para disposição retangular e triangular, em condições nulas de vento

de distribuição de água dos aspersores, comportamento este de acordo com o previsto por Chen \& Wallender (1984).

Nas superfícies de resposta (Figura 4) relativas ao espaçamento triangular entre aspersores pode-se, entretanto, observar que esta simetria foi desfeita fato também observado por Chen \& Wallender (1984). Diferentemente das disposições retangulares entre aspersores, nas disposições triangulares, o espaçamento entre linhas laterais $(\mathrm{Sp})$, na linha principal, não é equivalente ao espaçamento entre aspersores na linha lateral (Sl). Desta forma, os vazios abertos em função do aumento do espaçamento entre aspersores na Sl podem ser cobertos quando o espaçamento entre linhas forem menores que $40 \%$ do diâmetro molhado pelo aspersor (Figura 4).

Coeficientes de uniformidade de Christiansen (CUC) simulados em diferentes combinações de três velocidades de vento $\left(1,4,2,8\right.$ e $\left.4,2 \mathrm{~m} \mathrm{~s}^{-1}\right)$ e três direções predominantes de incidência do vento (perpendicular à linha lateral de aspersores, $45^{\circ}$ em relação à linha lateral de aspersores e paralelo à linha lateral de aspersores) em função do espaçamento (\% DM) entre canhões Plona-RL250 em disposição retangular entre aspersores, foram obtidos para os perfis típicos II, III e IV de distribuição de água determinados na análise de agrupamento, Figuras 5, 6 e 7, respectivamente.

Observa-se, nas Figuras 5, 6 e 7 que, de maneira geral, ao se comparar as três superfícies de resposta situadas em uma mesma linha (mesma velocidade de vento), tem-se uma pequena

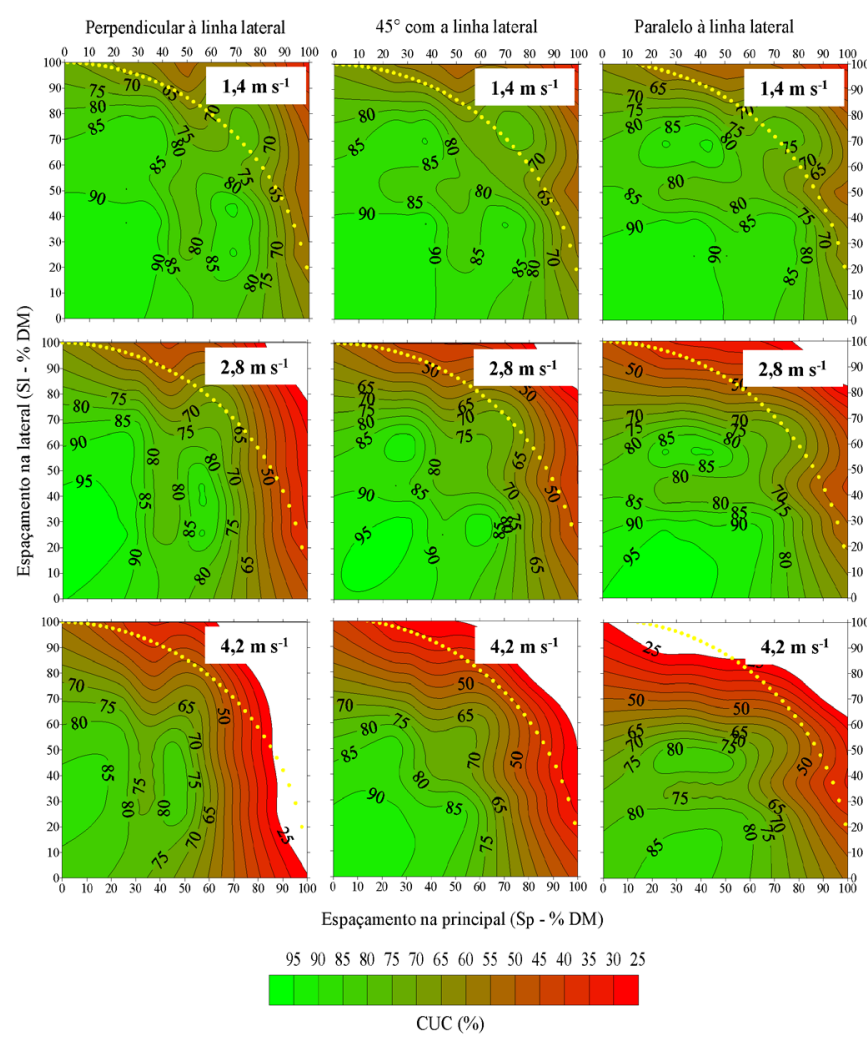

Figura 5. Valores de coeficiente de Christiansen - CUC (\%) em função do espaçamento entre aspersores (\% DM), para disposição retangular entre aspersores (Perfil típico II do canhão Plona-RL250)

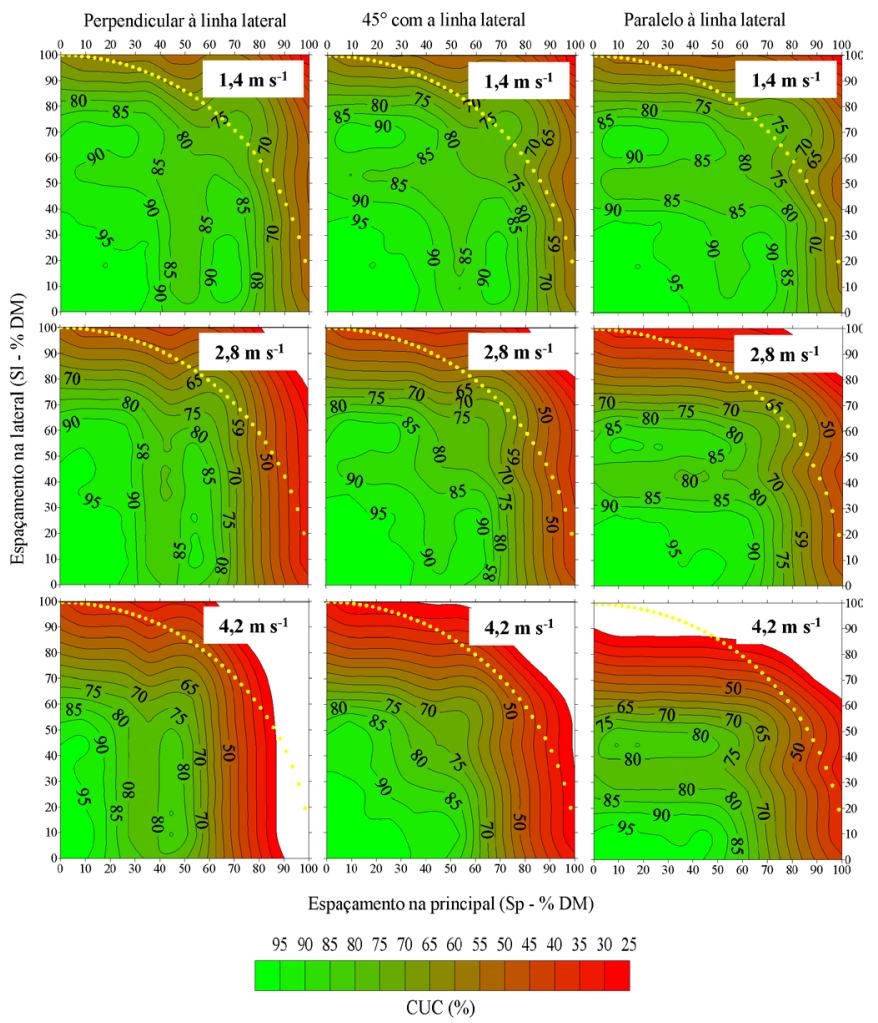

Figura 6. Valores de coeficiente de Christiansen - CUC (\%) em função do espaçamento entre aspersores (\% DM), para disposição retangular entre aspersores (Perfil típico III do canhão Plona-RL250) 


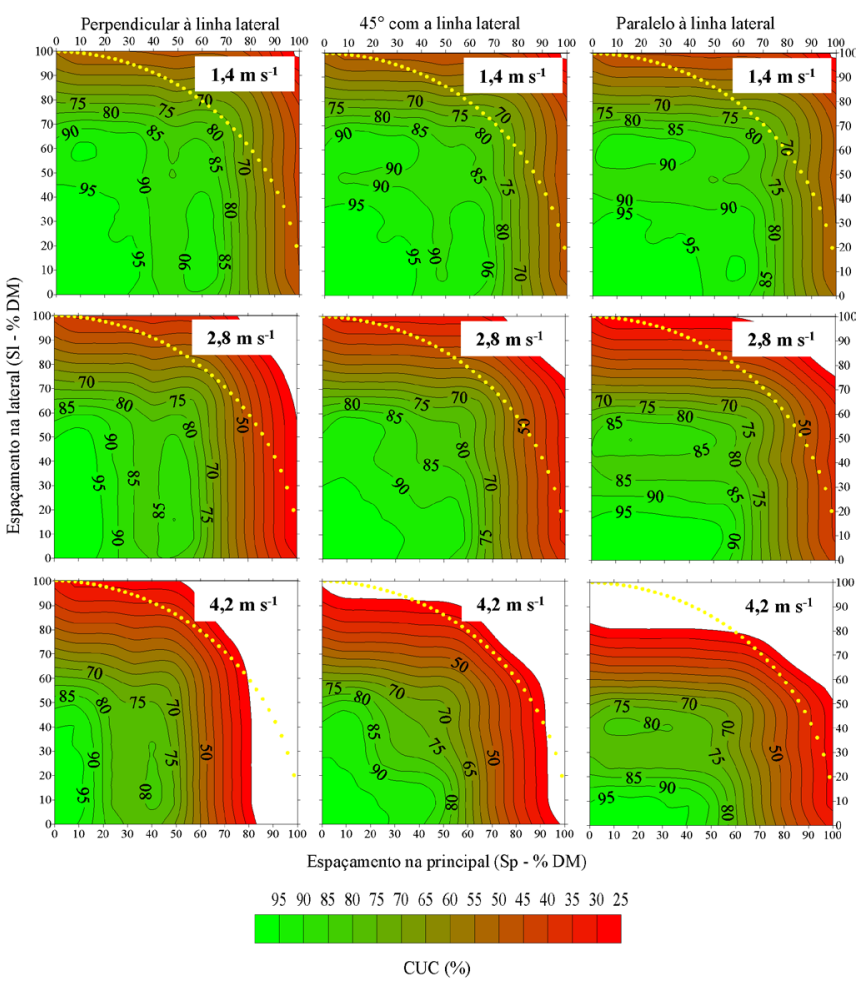

Figura 7. Valores de coeficiente de Christiansen - CUC (\%) em função do espaçamento entre aspersores (\% DM), para disposição retangular entre aspersores (Perfil típico IV do canhão Plona-RL250)

variação dos valores de CUC, que reflete em uma pequena influência da direção do vento sobre uniformidade; no entanto, cabe ressaltar que as superfícies situadas na extremidade direita das Figuras 5, 6 e 7, que representa uma condição na qual o vento incide na direção paralela às linhas laterais, apresentam maiores prejuízos nos valores de CUC enquanto as superfícies de respostas situadas na extremidade esquerda dessas figuras, que representam uma condição na qual o vento incide na direção perpendicular às linhas laterais, apresentam menores prejuízos na uniformidade, comportamento que está de acordo com o previsto por Shull \& Dylla (1976), Azevedo et al. (2000) e Bernardo et al. (2006) ao relatar que a melhor situação é aquela que permite uma incidência perpendicular do vento em relação às linhas laterais de aspersores.

Os resultados indicados nessas figuras demonstram que, para se obter valores de CUC próximo de $80 \%$, é necessário reduzir o espaçamento entre aspersores sempre que a velocidade do vento aumenta.

Para velocidades de vento até $1,4 \mathrm{~m} \mathrm{~s}^{-1}$ observam-se valores de CUC próximos de $80 \%$ com espaçamento entre aspersores de $40 \times 80 \%$ do diâmetro molhado, confirmando os limites recomendados por Keller \& Bliesner (1990) para os perfis D e E de Christiansen. Com velocidades de vento superiores a $1,4 \mathrm{~m} \mathrm{~s}^{-1}$ as simulações obtidas neste estudo demonstram que, para manter o mesmo nível de uniformidade $(\mathrm{CUC}=80 \%)$ e o mesmo espaçamento $\mathrm{Sl}(40 \% \mathrm{DM})$ é conveniente reduzir o espaçamento Sp entre 10 a $15 \%$ para cada incremento de 1,4 $\mathrm{m} \mathrm{s}^{-1}$ na velocidade do vento.

Nas Figuras 8, 9 e 10 são apresentadas superfícies de resposta dos coeficientes de uniformidade de Christiansen

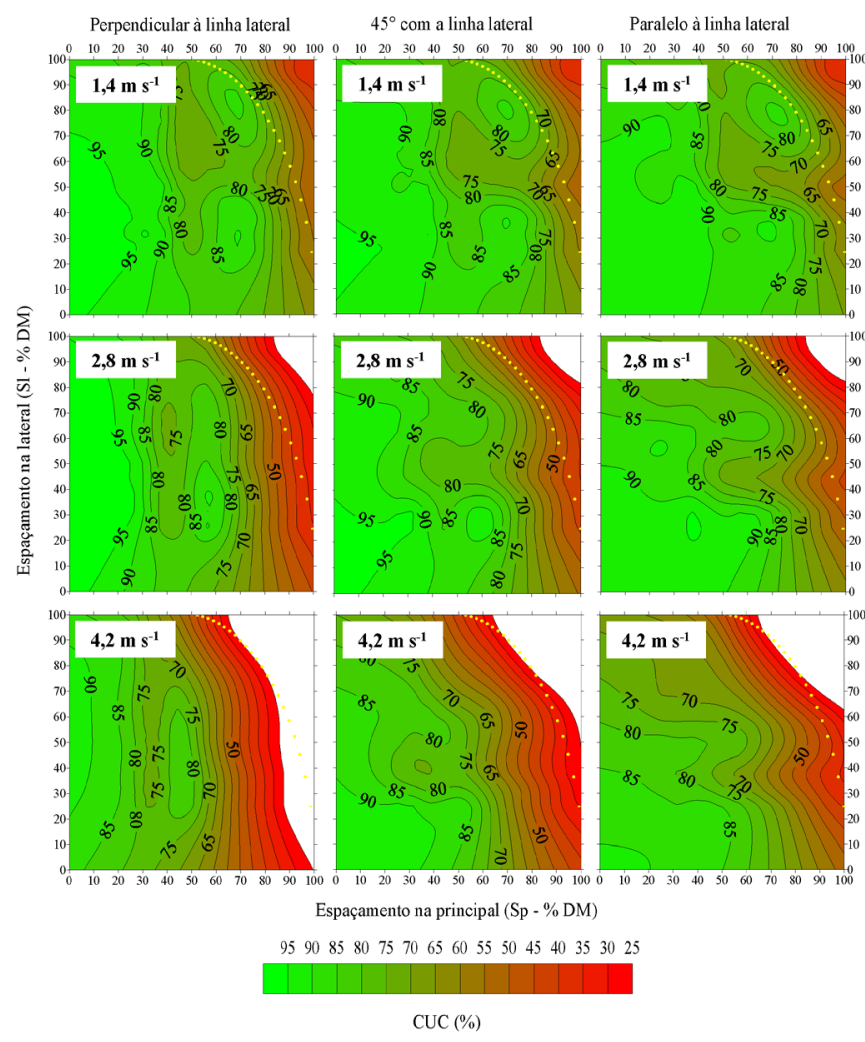

Figura 8. Valores de coeficiente de Christiansen - CUC (\%) em função do espaçamento entre aspersores (\% DM), para disposição triangular entre aspersores (Perfil típico II do canhão Plona-RL250)

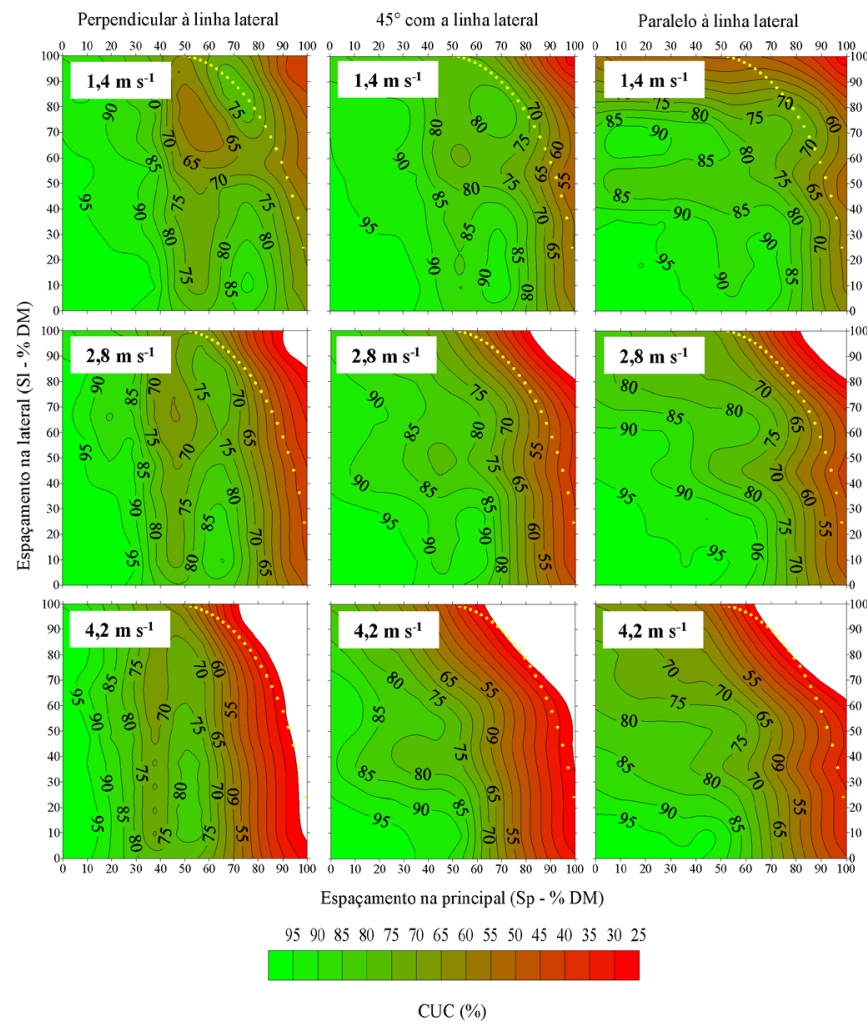

Figura 9. Valores de coeficiente de Christiansen - CUC (\%) em função do espaçamento entre aspersores (\% DM), para disposição triangular entre aspersores (Perfil típico III do canhão Plona-RL250) 


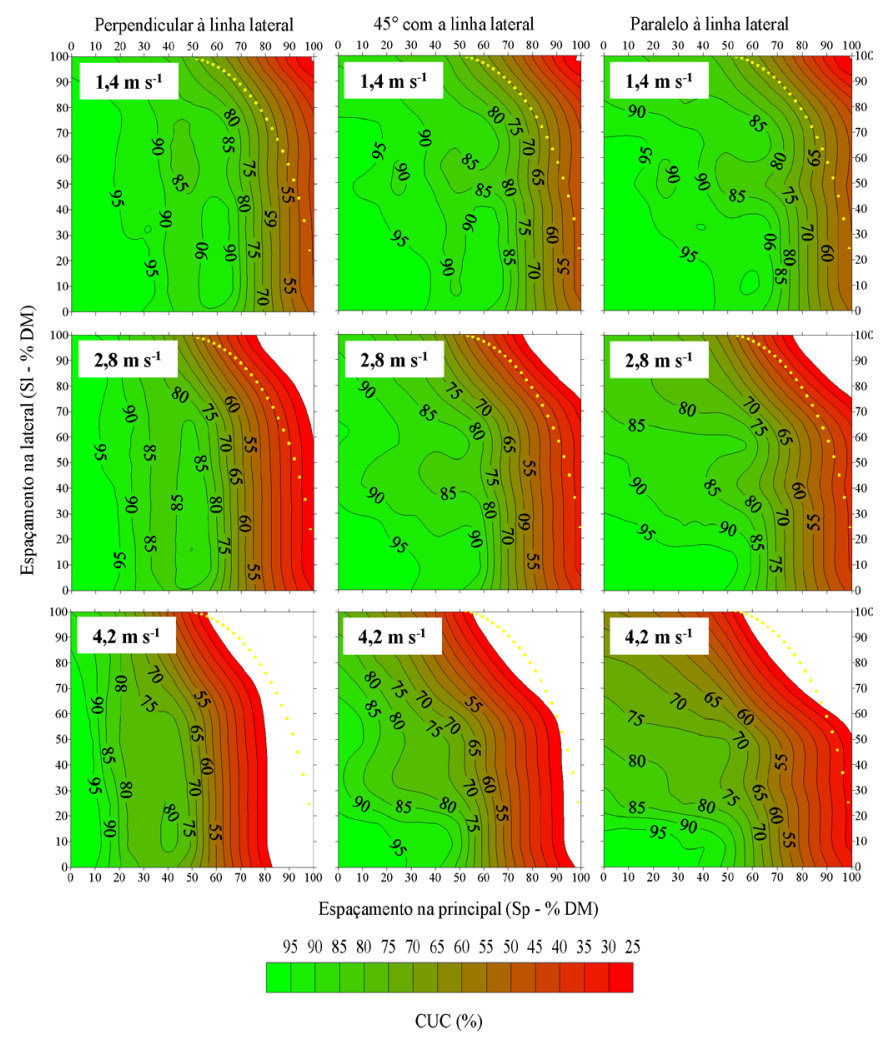

Figura 10. Valores de coeficiente de Christiansen CUC (\%) em função do espaçamento entre aspersores (\% DM), para disposição triangular entre aspersores (Perfil típico IV do canhão Plona-RL250)

(CUC) correspondentes aos perfis típicos II, III e IV do canhão Plona-RL250, em disposição triangular, nas mesmas condições de velocidade de vento avaliadas para os espaçamentos retangulares e as mesmas três direções de vento predominantes.

Observa-se, com a incidência do vento na direção perpendicular à linha lateral de aspersores (coluna da esquerda) que valores de CUC próximos de $80 \%$ são obtidos, em geral, quando os espaçamentos Sp não excedem 75, 60 e 40\% do diâmetro molhado para as velocidades de vento de 1,4;2,8 e $4,2 \mathrm{~m} \mathrm{~s}^{-1}$, respectivamente. Com a incidência da direção do vento de $45^{\circ} \mathrm{em}$ relação à linha lateral, esses limites passam a ser, respectivamente, 75,50 e $30 \%$ do diâmetro molhado para as mesmas velocidades de vento. Com a incidência do vento na direção paralela à linha lateral de aspersores (coluna da direita) para velocidades superiores a $2,8 \mathrm{~m} \mathrm{~s}^{-1}$, a região de CUC maior que $80 \%$ depende tanto de Sp quanto do Sl.

A comparação entre valores de CUC (\%) para disposição triangular e retangular de aspersores indica que, de maneira geral, para as mesmas condições de vento quando $\mathrm{Sl}>\mathrm{Sp}$, os valores de CUC obtidos em arranjos triangulares são superiores àqueles obtidos em arranjos retangulares; mesmo assim, para as mesmas condições de vento, quando $\mathrm{Sp}>\mathrm{Sl}$ (situação prática mais desejada) praticamente não existem diferenças entre valores de CUC obtidos com os arranjos triangulares e retangulares.

É importante ressaltar ainda que, ao empregar espaçamentos entre aspersores ( $\mathrm{Sl} \mathrm{e} \mathrm{Sp}$ ) inferiores a 40\% do diâmetro molhado, níveis desejáveis de uniformidades são atingidos para todas as velocidades de vento estudadas independente do posicionamento da linha lateral em relação à direção do vento e da disposição dos aspersores no campo, estando este comportamento de acordo com o previsto por Keller \& Bliesner (1990).

\section{Conclusões}

1. A direção do vento exerce pouca influência sobre os valores de coeficiente de Christiansen - CUC obtidos com auxílio da simulação computacional.

2. Na comparação entre disposições retangulares e triangulares de aspersores observaram-se, mediante simulação computacional, melhores valores na uniformidade de distribuição de água somente quando o espaçamento entre aspersores na linha lateral (Sl) foi maior que o espaçamento entre linhas laterais ao longo da linha principal (Sp).

\section{Literatura Citada}

Azevedo, H. J.; Bernardo, S.; Ramos, M. M.; Sediyama, G. C.; Cecon, P. R. Influência de fatores climáticos e operacionais sobre a uniformidade de distribuição de água, em um sistema de irrigação por aspersão de alta pressão. Revista Brasileira de Engenharia Agrícola e Ambiental, v.4, p.152-158, 2000.

Bernardo, S.; Soares, A. A.; Mantovani, E. C. Manual de irrigação. 8.ed. Viçosa: UFV, 2006. 625p.

Chen, D.; Wallender, W. W. Economic sprinkler selection, spacing and orientation. Transactions of the American Society of Agricultural Engineers, v.27, p.737-743, 1984.

Christiansen, J. E. Irrigation by sprinkling. Berkeley: University of California, 1942. 124p. Bulletin 670

Colombo, A.; Prado, G.; Oliveira, H. F. E. Modeling wind effects on travelling gun sprinkler water application uniformity. ASABE Annual International Meeting, 2009, Reno. Proceedings... Nevada: ASABE, 2009. CD Rom

Faria, L. C. Simulação da uniformidade de aplicação de água em sistemas convencionais de irrigação operando sob diferentes condições de vento. Lavras: UFLA, 2008. 76p. Dissertação Mestrado

Faria, L. C.; Colombo, A.; Oliveira, H. F. E. de; Prado, G. Simulação da uniformidade da irrigação de sistemas convencionais de aspersão operando sob diferentes condições de vento. Engenharia Agrícola, v.29, p.19-27, 2009.

Keller, J.; Bliesner, R. D. Sprinkle and trickle irrigation. New York: AnaviBook/Van Nostrand Reinhold, 1990. 652p.

Prado, G.; Colombo A. Distribuição espacial de água aplicada por equipamentos autopropelidos de irrigação - Parte I: Modelagem com o Simulasoft. Irriga, v.15, p.51-62, 2010a.

Prado, G.; Colombo A. Distribuição espacial de água aplicada por equipamentos autopropelidos de irrigação - Parte II: Validação do Simulasoft. Irriga, v.15, p.63-74, 2010 b.

Prado, G.; Colombo, A.; Barreto, A. C.; Matos, F. A. de; Ferreira Júnior, J. J. Uniformidade de aplicação de água pelo aspersor PLONA-RL250 em sistemas estacionários de irrigação. Irriga, v.13, p.220-234, 2008.

Richards, P. J.; Weatherhead, E. K. Prediction of raingun application patterns in windy conditions. Journal of Agricultural Engineering Research, v.54, p.281-291, 1993. 
Seginer, I.; Kantz, D.; Nir D. The distortion by wind of the distribution patterns of single sprinklers. Agricultural Water Management, v.19, p.341-359. 1991.

Shull, H.; Dylla, A. S. Wind effects on water application patterns from a large, single nozzle sprinkler. Transactions of the American Society of Agricultural Engineers, v.19, p.501-504,1976.

Smith, R.J.; Gillies, M. H.; Newell, G.; Foley, J. P. A decision support model for travelling gun irrigation machines, Biosystems Engineering, v.100, p.126-136, 2008.

Solomon, K.; Bezdek, J. C. Characterizing sprinkler distribution patterns with a clustering algorithm. Transactions of the American Society of Agricultural Engineers, v.23, p.899906, 1980.
Tarjuelo, J. M.; Monteiro, J.; Carrion, P. A.; Honrubia, F. T.; Calvo, M. A. Irrigation uniformity with medium size sprinklers part II: Influence of wind and other factors on water distribution. Transactions of the American Society of Agricultural Engineers, v.42, p.677-689, 1999.

Tou, J.; Gonzales, R. C. Pattern recognition principle. London: Addoson-Wesley, 1974. 377p.

Vellame, L. M.; Souza, W. de J.; Armindo, R. A.; Coelho, R. D. Determinação do perfil radial e da uniformidade do aspersor modelo Komet 140 Plus operando em sistema autopropelido. Revista Brasileira de Agricultura Irrigada v.5, p.105-112, 2011.

Vories, E. D.; Bernuth, R. D. von. Single nozzle sprinkler performance in wind. Transactions of the American Society of Agricultural Engineers, v.29, p.1325-1330, 1986. 